\title{
Fundamental Domains for Rhombic Lattices with Dihedral Symmetry of Order 8
}

\author{
Joseph Ray Clarence G. Damasco \\ Institute of Mathematics, College of Science \\ University of the Philippines Diliman \\ Quezon City, Philippines \\ jrcgdamasco@math.upd.edu.ph
}

\author{
Dirk Frettlöh \\ Technische Fakultät \\ Universität Bielefeld \\ Bielefeld, Germany
}

dfrettloeh@techfak.uni-bielefeld.de

\author{
Manuel Joseph C. Loquias \\ Institute of Mathematics, College of Science \\ University of the Philippines Diliman \\ Quezon City, Philippines \\ mjcloquias@math.upd.edu.ph
}

Submitted: Apr 18, 2018; Accepted: May 24, 2019; Published: Jul 5, 2019

(C) The authors. Released under the CC BY-ND license (International 4.0).

\begin{abstract}
We show by construction that every rhombic lattice $\Gamma$ in $\mathbb{R}^{2}$ has a fundamental domain whose symmetry group contains the point group of $\Gamma$ as a subgroup of index 2. This solves the last open case of a question raised in a preprint by the authors on fundamental domains for planar lattices whose symmetry groups properly contain the point groups of the lattices.
\end{abstract}

Mathematics Subject Classifications: 52C05, 52C20, 05B 45

\section{Introduction}

A lattice $\Gamma$ in $\mathbb{R}^{d}$ is the $\mathbb{Z}$-span of $d$ linearly independent vectors in $\mathbb{R}^{d}$. The lattice $\Gamma$ forms a group that is isomorphic to the free abelian group of rank $d$. The point group $P(\Gamma)$ of $\Gamma$ is the set of Euclidean isometries in $\mathbb{R}^{d}$ fixing both $\Gamma$ and the origin. That is, $P(\Gamma)$ is a subgroup of the orthogonal group $O(d)$ consisting of those transformations that fix $\Gamma$. A fundamental domain for $\Gamma$ is a complete set of representatives of the orbits of $\mathbb{R}^{d}$ under the action of the group $\Gamma$. For example, $F=\left[-\frac{1}{2}, \frac{1}{2}\right)^{2}$ is a fundamental domain for the lattice $\Gamma=\mathbb{Z}^{2} \subseteq \mathbb{R}^{2}$. We are interested in the geometric properties of fundamental domains, in particular, their symmetries. For a given $X \subseteq \mathbb{R}^{d}$, the symmetry group $\operatorname{Sym}(X)$ of $X$ is the group of Euclidean isometries in $\mathbb{R}^{d}$ that leave $X$ invariant. In 
our example, $\operatorname{Sym}(F)$ consists of the identity and the reflection in the line $y=x$. Now, $F$ differs from the square region $Q=\left[-\frac{1}{2}, \frac{1}{2}\right]^{2}$ only by a set of Lebesgue measure zero, and $Q$ is more symmetric compared to $F$ with $\operatorname{Sym}(Q)=P(\Gamma)$. Note that throughout this paper, Lebesgue measure is used.

In order to discuss symmetries or geometric properties of fundamental domains, it is convenient to allow repetitions of representatives on sets of measure zero. In particular, we restrict ourselves to consider compact fundamental domains. Thus, in this paper, a fundamental domain for a lattice $\Gamma$ is a compact set $F$ such that (i) the Minkowski sum $F+\Gamma=\{p+\mathbf{v} \mid p \in F, \mathbf{v} \in \Gamma\}$ equals $\mathbb{R}^{d}$ and (ii) for any nonzero $\mathbf{v} \in \Gamma, \operatorname{int}(F) \cap \operatorname{int}(F+\mathbf{v})=\varnothing$.

The following fact generalizes the earlier example: For any lattice $\Gamma$, the Voronoi region $V$ of the origin is a fundamental domain for $\Gamma$ such that $\operatorname{Sym}(V)$ equals $P(\Gamma)$ [3]. Recall that the Voronoi region of a point $\mathbf{v}$ of a lattice $\Gamma$ in $\mathbb{R}^{d}$ is the collection of points in $\mathbb{R}^{d}$ closest to $\mathbf{v}$ than to any other point in $\Gamma$. The following question then naturally arises: Is there a fundamental domain $F$ for $\Gamma$ that has a symmetry group larger than $P(\Gamma)$, in the sense that $\operatorname{Sym}(F)$ properly contains $P(\Gamma)$ ?

About the year 2000 Veit Elser constructed a fundamental domain for $\mathbb{Z}^{2}$ exhibiting eightfold dihedral symmetry rather than the expected fourfold dihedral symmetry and a fundamental domain for the hexagonal lattice in $\mathbb{R}^{2}$ possessing twelvefold dihedral symmetry $[6,5]$. Interestingly, this latter fundamental domain appears in different contexts in [1] and [2], where it serves as a window and an atomic surface, respectively, for mathematical quasicrystals.

The procedures in [5] were refined and generalized in [3] to obtain fundamental domains for rectangular lattices having the symmetries of a square. It was also shown that for oblique lattices, one can use a rectangular fundamental domain with one edge length equal to that of a basis vector, and a suitably chosen height. In fact, for a certain subclass of oblique lattices, this rectangular fundamental domain may be a square. Thus, if a bound for $[\operatorname{Sym}(F): P(\Gamma)]$ for planar lattices exists, it must be at least 4 .

Summarizing, for each planar lattice $\Gamma$ mentioned so far, there is a fundamental domain $F$ for $\Gamma$ for which $[\operatorname{Sym}(F): P(\Gamma)]=2$ (Theorem 1.1 in [3]). Hence, the only missing case for planar lattices is that of rhombic lattices and this paper is dedicated to fill this gap.

A rhombic lattice is a planar lattice for which there exists a basis consisting of two vectors of equal length such that the angle between the vectors is not $\frac{\pi}{2}$ (square lattice), or $\frac{\pi}{3}$ or $\frac{2 \pi}{3}$ (hexagonal lattice). For any rhombic lattice $\Gamma, P(\Gamma)$ is isomorphic to the dihedral group $D_{2}$ of order 4 . In this paper, we construct for each rhombic lattice a fundamental domain $F$ with fourfold dihedral symmetry. Some of these fundamental domains are illustrated in Figures 4 and 10. Our procedure differs substantially from the methods used in [3], as these methods do not seem to be applicable to rhombic lattices. Whereas the constructions in [3] involve removing subregions and adding different ones at each step, the construction we employ here simply adds regions at each step.

We prove our main result (Theorem 1) as follows: First, we prove that it is enough to consider rhombic lattices whose diagonals have ratio at most 3 (Lemma 2). We then show that in order to construct $F$ it suffices to select a suitable subregion of a rectangle that satisfies certain conditions (see Rh1-Rh3 below). It turns out from Lemma 4 that 
it is enough to consider rectangles with edge ratio at most 3 . We then establish in Lemma 7 that after taking care of a particular subregion of the rectangle, the remaining portion of the rectangle may be dealt with by considering a smaller rectangle and finding a corresponding subregion satisfying analogues of conditions Rh1-Rh3, thereby creating an iterative process. Finally, we argue that the limit obtained by this iteration gives rise to a fundamental domain for $\Gamma$ satisfying the desired properties.

\section{Main result}

Theorem 1. Let $\Gamma$ be a rhombic lattice. Then there exists a fundamental domain $F$ for $\Gamma$ such that $[\operatorname{Sym}(F): P(\Gamma)]=2$, in particular, $\operatorname{Sym}(F) \cong D_{4}$.

Proof. Let $\Gamma$ be a rhombic lattice. Because the properties we will be dealing with are invariant under similarities, we assume without loss of generality that $\Gamma$ has basis $\left\{\left(\begin{array}{c}2 m \\ 0\end{array}\right),\left(\begin{array}{c}m \\ n\end{array}\right)\right\}$, where $0<m<n$. Here, a basis for $\Gamma$ consisting of vectors of equal length is $\left\{\left(\begin{array}{c}m \\ -n\end{array}\right),\left(\begin{array}{c}m \\ n\end{array}\right)\right\}$. Let $\alpha$ be the reflection in the $y$-axis and $\beta$ be the half-turn about the origin (or central symmetry in $\mathbb{R}^{2}$ ). Then, $P(\Gamma)=\langle\alpha, \beta\rangle \cong D_{2}$.

Suppose first that $n \leq 3 m$. Let $\mathcal{Q}$ be the triangular region with vertices $\left(\begin{array}{l}0 \\ 0\end{array}\right), X=\left(\begin{array}{c}m \\ 0\end{array}\right)$, and $W=\left(\begin{array}{l}0 \\ m\end{array}\right)$. The union $\mathcal{S}$ of the images of $\mathcal{Q}$ under $P(\Gamma)$ forms a region with symmetry group $D_{4}$. Translates of $\mathcal{S}$ by distinct vectors in $\Gamma$ are either disjoint or intersect on sets of measure zero. See Figure 1.

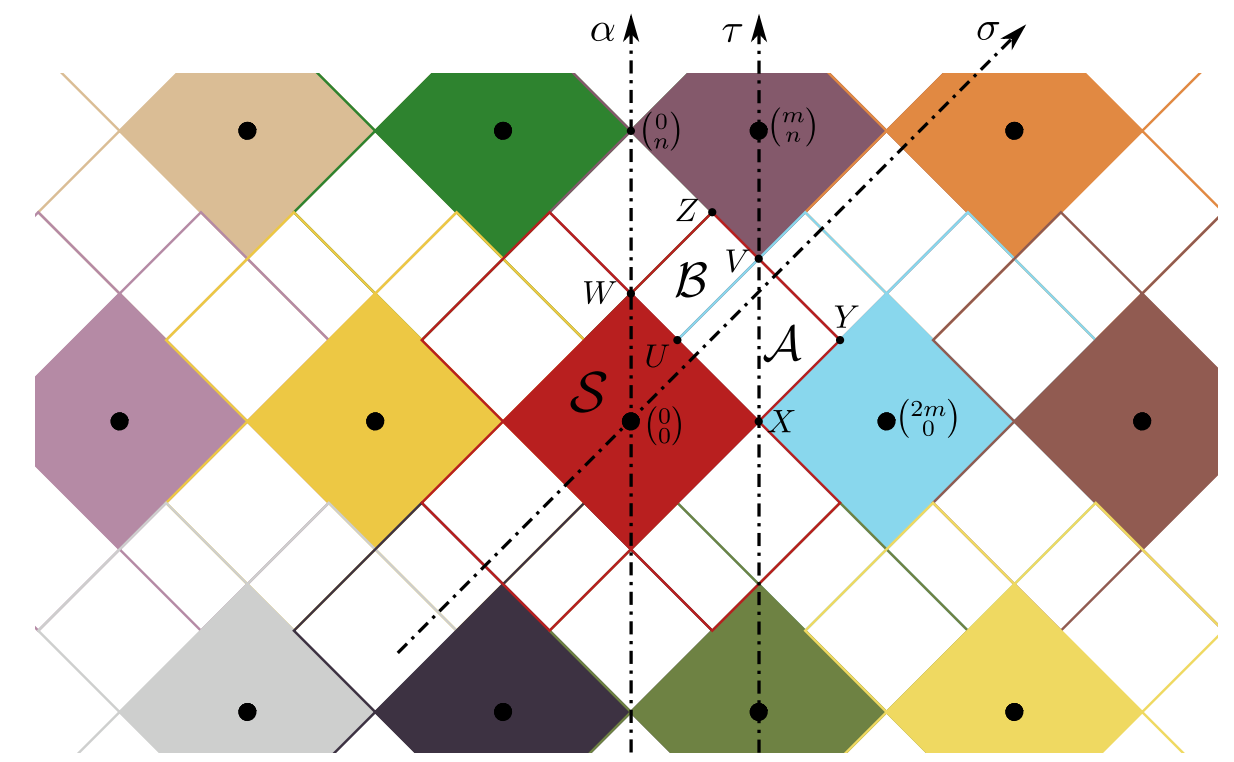

Figure 1: First step in constructing a highly symmetric fundamental domain for a rhombic lattice.

Now, consider the rectangular region $\mathcal{R}$ with vertices $W, X, Y$, and $Z$, where $Y Z$ lies on the line through $\left(\begin{array}{l}0 \\ n\end{array}\right)$ and $V=\left(\begin{array}{c}m \\ n-m\end{array}\right)$. We claim that there is a subset of $\mathcal{R}$ such that 
if $\mathcal{E}$ is the union of the images of this subset under $P(\Gamma)$, then the closure $F=\operatorname{cl}(\mathcal{S} \cup \mathcal{E})$ is a fundamental domain for $\Gamma$. If in addition the subset of $\mathcal{R}$ is preserved by the mirror reflection $\sigma$ in the perpendicular bisector of $W X$, then $F$ has symmetry group $\langle\alpha, \sigma\rangle \cong D_{4}$.

Since $n \leq 3 m$, the ratio $\frac{W X}{X Y}=\frac{2 m}{(n-m)}$ is at least 1. Partition $\mathcal{R}$ into the two sets: $\mathcal{A}$, the square $X Y V U$, and $\mathcal{B}=\operatorname{cl}(\mathcal{R} \backslash \mathcal{A}$ ), the rectangle $W U V Z$ (see Figure 1). Note that if $n=3 m$, then $V=Z$ and $\mathcal{B}$ is empty.

Consider the images of $\mathcal{A}$ and $\mathcal{B}$ under $P(\Gamma)$ and their translates by $\Gamma$. Equivalently, the union of these images and translates give $\operatorname{Sym}(\Gamma)(\mathcal{A} \cup \mathcal{B})$. The only element of $\operatorname{Sym}(\Gamma) \mathcal{A}$ that overlaps with $\mathcal{A}$ is $\alpha(\mathcal{A})+\left(\begin{array}{c}2 m \\ 0\end{array}\right)$. In fact, the two are equal. Similarly, $\mathcal{B}=\beta(\mathcal{B})+\left(\begin{array}{c}m \\ n\end{array}\right)$, and $\mathcal{B}$ is disjoint with its other copies in $\operatorname{Sym}(\Gamma) \mathcal{B}$. Thus, it suffices to find closed sets $\mathcal{K} \subseteq \mathcal{A}$ and $\mathcal{L} \subseteq \mathcal{B}$ such that $\mathcal{K} \bullet\left(\alpha(\mathcal{K})+\left(\begin{array}{c}2 m \\ 0\end{array}\right)\right)=\mathcal{A}, \mathcal{L} \bullet\left(\beta(\mathcal{L})+\left(\begin{array}{c}m \\ n\end{array}\right)\right)=\mathcal{B}$, and $\mathcal{K} \cup \mathcal{L}$ is invariant under $\sigma$, where $\bullet$ denotes a union of sets whose intersection has measure zero. We take the aforementioned $\mathcal{E}$ to be the union of the images of $\mathcal{K} \cup \mathcal{L}$ under $P(\Gamma)$.

The required conditions above can also be expressed in terms of symmetries of $\mathcal{A}$ and $\mathcal{B}$. Denote by $T_{\mathbf{v}}$ the translation by the vector $\mathbf{v}$. Let $\tau=T_{\left(\begin{array}{c}m \\ 0\end{array}\right)} \alpha T_{\left(\begin{array}{c}m \\ 0\end{array}\right)}^{-1}$ and $\rho=T_{\frac{1}{2}\left(\begin{array}{c}m \\ n\end{array}\right)} \beta T_{\frac{1}{2}\left(\begin{array}{c}m \\ n\end{array}\right)}^{-1}$. That is, $\tau$ is the mirror reflection in $X V$ and $\rho$ is the half-turn about the center of $\mathcal{B}$ which is $\frac{1}{2}\left(\begin{array}{c}m \\ n\end{array}\right)$. Using the fact that for any vector $\mathbf{v}$ and orthogonal linear transformation $\psi$, $\psi T_{\mathbf{v}}=T_{\psi(\mathbf{v})} \psi$ holds, we find that indeed, $\tau=T_{\left(\begin{array}{c}2 m \\ 0\end{array}\right)} \alpha$ and $\rho=T_{\left(\begin{array}{c}m \\ n\end{array}\right)} \beta$. Thus, our main problem reduces to finding $\mathcal{K} \subseteq \mathcal{A}$ and $\mathcal{L} \subseteq \mathcal{B}$ satisfying the following properties.

Rh1. $\mathcal{K} \cup \tau(\mathcal{K})=\mathcal{A}$

Rh2. $\mathcal{L} \cup \rho(\mathcal{L})=\mathcal{B}$

Rh3. $\sigma(\mathcal{K} \bullet \mathcal{L})=\mathcal{K} \bullet \mathcal{L}$

In the degenerate case $n=3 m$, since $\mathcal{B}=\varnothing$, the problem reduces to finding $\mathcal{K} \subseteq \mathcal{A}$ that satisfies Rh1 and that is invariant under $\sigma$.

We now argue that the case where the ratio $\frac{n}{m}$ of the diagonals is greater than 3 may be dealt with similarly.

Lemma 2. It suffices to consider the case when $n \leq 3 m$.

Proof of Lemma 2. If $n>3 m$, let $t$ be the largest integer such that $n-2 m t>m$. For each $i$ from 1 to $t$, construct the square $[m(i-1), m i] \times[m(i-1), m i]$. Obtain the union $\mathcal{C}$ of the images of these squares under $P(\Gamma)$. We note that $\operatorname{Sym}(\mathcal{C}) \cong D_{4}$. By the choice of $t, \mathcal{C}$ does not intersect with any of its translates by non-horizontal vectors in $\Gamma$. See for example the squares in Figure 2(a), where $t=3$ and $\mathcal{C}$ is the union of the red squares. Note that the encircled dots represent lattice points. The points not encircled mark the furthest corners of the $\Gamma$-translates of $\mathcal{C}$.

Furthermore, $\mathcal{C}$ does not overlap with its horizontal $\Gamma$-translates because the squares of the form $[m(i-1+2 k), m(i+2 k)] \times[m(i-1), m i]$, where $k \in \mathbb{Z}$, are precisely the horizontal $\Gamma$-translates of $[m(i-1), m i] \times[m(i-1), m i]$, while those of the form $[m(i+2 k), m(i+1+$ $2 k)] \times[m(i-1), m i]$ are the horizontal $\Gamma$-translates of $[m(-i), m(-i+1)] \times[m(i-1), m i]=$ 


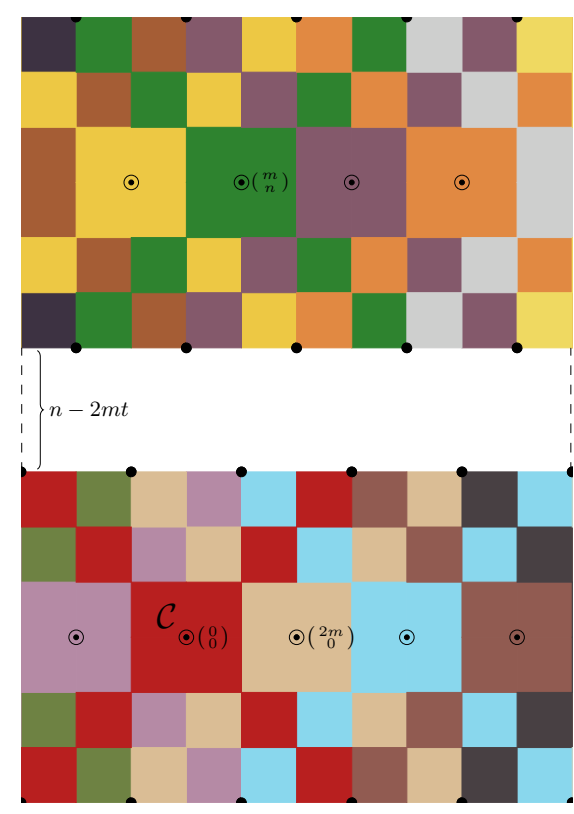

(a)

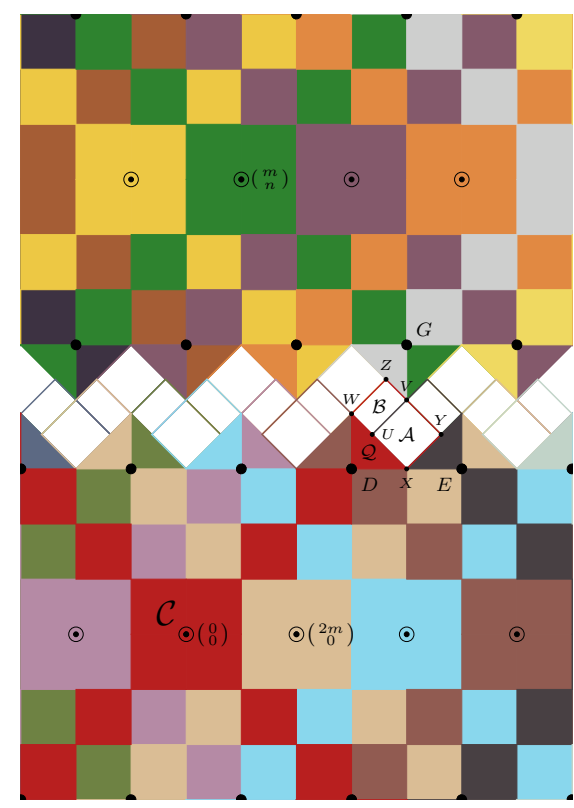

(b)

Figure 2: The case when the ratio of the diagonals is greater than 3. Here, the smaller encircled dots represent lattice points while larger dots represent the furthest corners of copies of $\mathcal{C}$.

$\alpha([m(i-1), m i] \times[m(i-1), m i])$. Thus, $\mathcal{C}$ does not overlap with its translates by nontrivial vectors in $\Gamma$. Moreover, the portion of the plane left uncovered by $\mathcal{C}+\Gamma$ is a union of horizontal strips of width $n-2 m t$.

Consider the points marked by dots on the boundary of one such horizontal strip, say the white strip in Figure 2(a). Each point on the lower boundary is both an upperright corner and an upper-left corner of two horizontally-separated copies of $\mathcal{C}$, while each vertex on the upper boundary is both a lower-right and lower-left corner of two horizontally-separated copies of $\mathcal{C}$. The upper layer of points is the translation of the lower layer by $\left(\begin{array}{c}m \\ n-2 m t\end{array}\right)$, and so the points on these two layers are spaced in the same way that the points of a rhombic lattice with basis $\left(\begin{array}{c}2 m \\ 0\end{array}\right)$ and $\left(\begin{array}{c}m \\ n-2 m t\end{array}\right)$ are spaced. Note that by the choice of $t, m<n-2 m t \leq 3 m$.

In Figure 2(b), $D=\left(\begin{array}{c}m t \\ m t\end{array}\right)$ is the upper-right corner of $\mathcal{C}, E=\left(\begin{array}{c}m(t+2) \\ m t\end{array}\right)$ is the upper-left corner of $\mathcal{C}+(t+1)\left(\begin{array}{c}2 m \\ 0\end{array}\right)$, and $G=\left(\begin{array}{c}m(t+1) \\ n-m t\end{array}\right)$ is the lower-left corner of $\mathcal{C}+\left(\begin{array}{c}m \\ n\end{array}\right)+t\left(\begin{array}{c}2 m \\ 0\end{array}\right)$. This time, let $\mathcal{Q}$ be the triangular region with vertices $D=\left(\begin{array}{c}m t \\ m t\end{array}\right), W=\left(\begin{array}{c}m t \\ m(t+1)\end{array}\right)$, and $X=\left(\begin{array}{c}m(t+1) \\ m t\end{array}\right)$. Since $m<n-2 m t, \mathcal{Q}$ lies entirely in the strip. Let $\mathcal{S}$ be the union of the images of $\mathcal{Q}$ under $P(\Gamma)$. Extending the argument used for $\mathcal{C}, \mathcal{S}$ does not overlap with its horizontal translates. Furthermore, because $m<n-2 m t, \mathcal{S}$ does not overlap with its non-horizontal

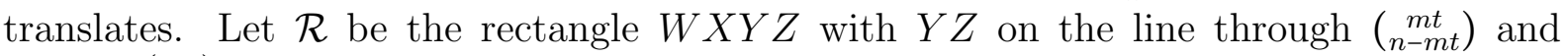
$V=\left(\begin{array}{c}m(t+1) \\ n-m(t+1)\end{array}\right)$. Again, we can partition $\mathcal{R}$ into $\mathcal{A}$, the square $X Y V U$, and $\mathcal{B}=\operatorname{cl}(\mathcal{R} \backslash \mathcal{A})$, the (possibly empty) rectangle $W U V Z$.

Consider the images of $\mathcal{A}$ and $\mathcal{B}$ under $P(\Gamma)$ and the translates of these by $\Gamma$. Similar 
to the case when $n \leq 3 m, \mathcal{A}=\alpha(\mathcal{A})+(t+1)\left(\begin{array}{c}2 m \\ 0\end{array}\right)$ and $\mathcal{B}=\beta(\mathcal{B})+\left(\begin{array}{c}m \\ n\end{array}\right)+t\left(\begin{array}{c}2 m \\ 0\end{array}\right)$. Thus, if there exist $\mathcal{K} \subseteq \mathcal{A}$ and $\mathcal{L} \subseteq \mathcal{B}$ satisfying conditions Rh1, Rh2, and Rh3, and $\mathcal{E}$ is formed accordingly, then the set $F=\operatorname{cl}(\mathcal{C} \cup \mathcal{S} \cup \mathcal{E})$ is a fundamental domain for $\Gamma$ with symmetry group $D_{4}$.

This confirms that it suffices to consider the case when the ratio $\frac{n}{m}$ of the diagonals of the rhombic lattice is at most 3 . In fact, we use the same $\mathcal{Q}, \mathcal{K}$, and $\mathcal{L}$ (up to similarity) for two rhombic lattices if the difference between their diagonal ratios is an even integer.

We now construct the sets $\mathcal{K}$ and $\mathcal{L}$, where we assume $1<\frac{n}{m} \leq 3$. Let $a$ and $b$, with $a \geq b$, be the edge lengths of $\mathcal{R}$ so that $\frac{2 m}{(n-m)}=\frac{a}{b}$. For convenience, we reorient $\mathcal{R}$ such that $U$ is at the origin, $\mathcal{A}=[0, b] \times[0, b]$, and $\mathcal{B}=[b-a, 0] \times[0, b]$ if $a \neq b$. Recall that $\mathcal{B}$ is empty when $a=b$, which happens when $n=3 \mathrm{~m}$. Recall also that $\sigma$ is the reflection in the perpendicular bisector of $W X, \tau$ is the reflection in the line through $X V$, and $\rho$ is the half-turn about the center of $\mathcal{B}$. In the present orientation of $\mathcal{R}$ the axis of $\sigma$ is vertical and the axis of $\tau$ has slope -1 .

Lemma 3. There exist $\mathcal{K}$ and $\mathcal{L}$ satisfying Rh1, Rh2, and Rh3 whenever the ratio $\frac{a}{b}$ is an integer.

Proof of Lemma 3. In each of the rectangles in Figure 3, the rightmost square is $\mathcal{A}$. For $\frac{a}{b}=1, \mathcal{B}$ is empty and it suffices to find $\mathcal{K} \subseteq \mathcal{A}=\mathcal{R}$ such that $\mathcal{K} \cup \tau(\mathcal{K})=\mathcal{A}$ and $\sigma(\mathcal{K})=\mathcal{K}$. One way to do this is to draw the two diagonals of $\mathcal{A}$ to divide it into four congruent triangles, and choose $\mathcal{K}$ to be the union of any two non-adjacent triangles, say the triangles marked red in Figure 3(a).

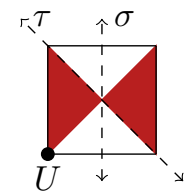

(a)

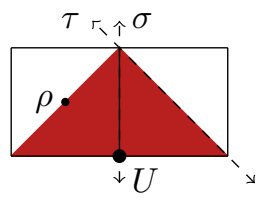

(b)

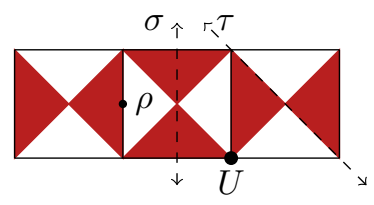

(c)

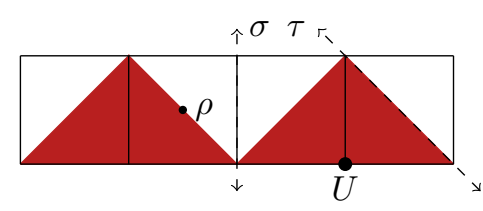

(d)

Figure 3: Choices for $\mathcal{K}$ and $\mathcal{L}$ when (a) $\frac{a}{b}=1$, (b) $\frac{a}{b}=2$, (c) $\frac{a}{b}=3$, (d) $\frac{a}{b}=4$.

If $\frac{a}{b}=2, \mathcal{B}$ is also a square, and $\mathcal{B}$ equals $\sigma(\mathcal{A})$. This together with Rh3 implies that $\sigma(\mathcal{K})$ must be $\mathcal{L}$. Note that the chosen $\mathcal{K}$ for $\frac{a}{b}=1$ cannot be used, because its image under $\sigma$ violates Rh2. Rather, one can take $\mathcal{K}$ to be one of the triangles into which the axis of $\tau$ divides $\mathcal{A}$, as illustrated in Figure 3(b).

We now consider the case when $\frac{a}{b} \geq 3$. Because $\frac{a}{b}$ is an integer, we can divide $\mathcal{R}$ into a row of $\frac{a}{b}$ congruent squares each of edge length $b$. If $\frac{a}{b}$ is odd, color triangles in each square as in the case when $\frac{a}{b}=1$, such that no two red triangles share a common edge (orientations of red triangles alternate). If $\frac{a}{b}$ is even, group the squares into adjacent pairs and color triangles in each pair as in the case when $\frac{a}{b}=2$. This is illustrated in Figures 3(c) and 3(d) for $\frac{a}{b}=3$ and $\frac{a}{b}=4$, respectively. In either case, if we let the red portion in $\mathcal{A}$ be $\mathcal{K}$ and the union of the other red portions be $\mathcal{L}$, then the sets $\mathcal{K}$ and $\mathcal{L}$ satisfy conditions Rh1, Rh2, and Rh3. 
Figure 4 shows the fundamental domains formed for the rhombic lattices with $\left(\begin{array}{c}m \\ n\end{array}\right)=\left(\begin{array}{l}3 \\ 5\end{array}\right)$ and $\left(\begin{array}{l}2 \\ 3\end{array}\right)$, which correspond to $\frac{a}{b}=3$ and 4 , respectively.

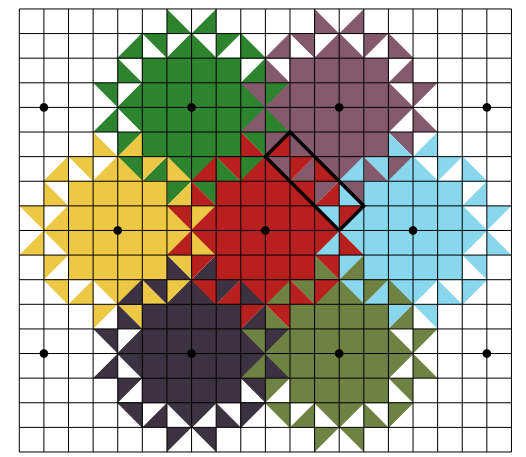

(a)

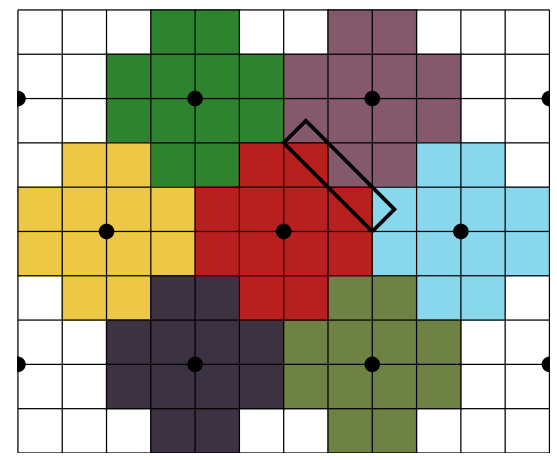

(b)

Figure 4: Some fundamental domains where (a) $\frac{a}{b}=3$ and (b) $\frac{a}{b}=4$.

We shall use the same idea for more general rectangles $\mathcal{R}$, that is, we subdivide $\mathcal{R}$ into squares and take suitable subregions. If the rectangle has irrational edge ratio, the number of squares is necessarily infinite [4]. The method we develop below works for any real value of the ratio, terminating after a finite number of steps precisely when the edge ratio is rational. First, we reduce the cases we have to consider.

Lemma 4. It suffices to construct $\mathcal{K}$ and $\mathcal{L}$ for rectangles $\mathcal{R}$ with edge ratio $\frac{a}{b}$ not exceeding 3 .

Proof of Lemma 4. Suppose $\mathcal{R}=\mathcal{A} \cup \mathcal{B}$ has edge ratio $\frac{a}{b} \geq 1$, and $\mathcal{K}$ and $\mathcal{L}$ satisfy $\operatorname{Rh} 1$, $\mathrm{Rh} 2$, and Rh3. To prove the lemma, we show that for $\mathcal{R}^{\prime}=\mathcal{A} \cup \mathcal{B}^{\prime}$ with edge ratio $\frac{a}{b}+2$ and corresponding $\rho^{\prime}$ and $\sigma^{\prime}$, one can construct $\mathcal{L}^{\prime} \subseteq \mathcal{B}^{\prime}$ from $\mathcal{K}$ and $\mathcal{L}$ such that $\mathcal{K}$ and $\mathcal{L}^{\prime}$ satisfy the corresponding analogues of Rh1, Rh2, and Rh3.

Note that $\tau(\mathcal{A})=\mathcal{A}, \rho(\mathcal{B})=\mathcal{B}, \sigma(\mathcal{A} \cup \mathcal{B})=\mathcal{A} \cup \mathcal{B}$. Refer to Figure 5. Consider the rectangle $\mathcal{R} \cup \rho(\mathcal{A})=\mathcal{A} \cup \mathcal{B} \cup \rho(\mathcal{A})$. We have

$$
\rho(\mathcal{A} \cup \mathcal{B} \bullet \rho(\mathcal{A}))=\mathcal{A} \bullet \mathcal{B} \sqcup \rho(\mathcal{A}) .
$$

Let $\sigma^{\prime}=\rho \sigma \rho^{-1}=\rho \sigma \rho$, that is, $\sigma^{\prime}$ is the reflection in the line formed by applying $\rho$ to the axis of $\sigma$. Then,

$$
\sigma^{\prime}(\mathcal{B} \cup \rho(\mathcal{A}))=\rho \sigma \rho(\mathcal{B} \cup \rho(\mathcal{A}))=\rho \sigma(\mathcal{B} \cup \mathcal{A})=\rho(\mathcal{B} \cup \mathcal{A})=\mathcal{B} \bullet \rho(\mathcal{A}) .
$$

Set $\mathcal{B}^{\prime}=\mathcal{B} \cup \rho(\mathcal{A}) \cup \sigma^{\prime}(\mathcal{A})$ and $\mathcal{R}^{\prime}=\mathcal{A} \cup \mathcal{B}^{\prime}$. Observe that the rectangle $\mathcal{R}^{\prime}$ has edges with ratio $\frac{a}{b}+2$. Let $\rho^{\prime}=\sigma^{\prime} \rho\left(\sigma^{\prime}\right)^{-1}=\sigma^{\prime} \rho \sigma^{\prime}$, that is, $\rho^{\prime}$ is the half-turn about the image of the center of $\rho$ under $\sigma^{\prime}$. It is easy to verify that

$$
\rho^{\prime}\left(\mathcal{B}^{\prime}\right)=\mathcal{B}^{\prime} \text { and } \sigma^{\prime}\left(\mathcal{R}^{\prime}\right)=\mathcal{R}^{\prime} .
$$

Set $\mathcal{L}^{\prime}=\mathcal{L} \cup \rho \tau(\mathcal{K}) \cup \sigma^{\prime}(\mathcal{K}) \subseteq \mathcal{B}^{\prime}$. We claim that

$$
\sigma^{\prime}\left(\mathcal{K} \cup \mathcal{L}^{\prime}\right)=\mathcal{K} \bullet \mathcal{L}^{\prime} \text { and } \mathcal{L}^{\prime} \bullet \rho^{\prime}\left(\mathcal{L}^{\prime}\right)=\mathcal{B}^{\prime}
$$




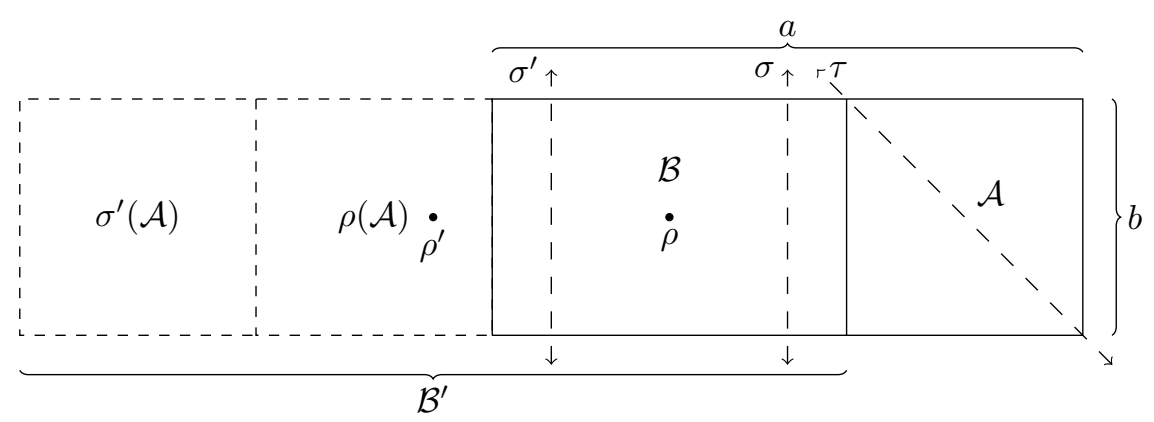

Figure 5: Constructing rectangle $\mathcal{R}^{\prime}$ from rectangle $\mathcal{R}$ with edge ratio increased by 2 .

First, by Rh1 and $\operatorname{Rh} 2, \mathcal{L} \cup \rho \tau(\mathcal{K})$ and $\rho(\mathcal{L} \cup \mathcal{K})$ partition $\mathcal{B} \bullet \rho(\mathcal{A})$, which is invariant under $\sigma^{\prime}$. Because $\sigma^{\prime} \rho(\mathcal{L} \cup \mathcal{K})=\rho \sigma(\mathcal{L} \bullet \mathcal{K})=\rho(\mathcal{L} \bullet \mathcal{K})$ by Rh3, we obtain that

$$
\sigma^{\prime}(\mathcal{L} \bullet \rho \tau(\mathcal{K}))=\mathcal{L} \bullet \rho \tau(\mathcal{K})
$$

It then follows that $\sigma^{\prime}\left(\mathcal{K} \cup \mathcal{L}^{\prime}\right)=\mathcal{K} \bullet \mathcal{L}^{\prime}$.

Finally, we deduce that

$$
\begin{aligned}
\mathcal{L}^{\prime} \bullet \rho^{\prime}\left(\mathcal{L}^{\prime}\right) & =\mathcal{L} \bullet \rho \tau(\mathcal{K}) \bullet \sigma^{\prime}(\mathcal{K}) \cup \sigma^{\prime} \rho \sigma^{\prime}\left(\mathcal{L} \bullet \rho \tau(\mathcal{K}) \bullet \sigma^{\prime}(\mathcal{K})\right) \\
& =\sigma^{\prime}(\mathcal{L} \bullet \rho \tau(\mathcal{K})) \cup \sigma^{\prime}(\mathcal{K}) \cup \sigma^{\prime} \rho(\mathcal{L} \bullet \rho \tau(\mathcal{K}) \bullet \mathcal{K}) \\
& =\sigma^{\prime}(\mathcal{A} \bullet \mathcal{B} \bullet \rho(\mathcal{A}))=\mathcal{B}^{\prime} .
\end{aligned}
$$

Thus, for $\mathcal{R}^{\prime}=\mathcal{A} \cup \mathcal{B}^{\prime}, \tau, \rho^{\prime}$, and $\sigma^{\prime}$, and $\mathcal{K} \subseteq \mathcal{A}$ and $\mathcal{L}^{\prime} \subseteq \mathcal{B}^{\prime}$ as above, the corresponding analogues of conditions Rh1, Rh2, and Rh3 are satisfied. This shows how one can obtain from a given construction for a given rectangle a corresponding construction for a rectangle with edge ratio increased by 2 . Hence, it does indeed suffice to consider those with edge ratio at most 3 .

Remark 5. In Lemma 4, the edge ratio $\frac{a}{b}$ may be an integer. The construction for the integer edge ratio case discussed in Lemma 3 is precisely what is obtained when the procedure in the proof of Lemma 4 is applied inductively starting with the constructions for $\frac{a}{b}=2$ and $\frac{a}{b}=3$.

The reasoning in the proof of Lemma 4 applies as well to subsets of $\mathcal{A}, \mathcal{B}, \mathcal{K}$, and $\mathcal{L}$ that satisfy analogous conditions. We note them in the following remark as they will be useful later.

Remark 6.

(a) Suppose $\mathcal{A}^{*} \subseteq \mathcal{A}$ and $\mathcal{B}^{*} \subseteq \mathcal{B}$ such that $\tau\left(\mathcal{A}^{*}\right)=\mathcal{A}^{*}, \rho\left(\mathcal{B}^{*}\right)=\mathcal{B}^{*}, \sigma\left(\mathcal{A}^{*} \cup \mathcal{B}^{*}\right)=\mathcal{A}^{*} \sqcup \mathcal{B}^{*}$. Then, $\mathcal{A}^{*} \cup \mathcal{B}^{*} \bullet \rho\left(\mathcal{A}^{*}\right) \cup \sigma^{\prime}\left(\mathcal{A}^{*}\right)$ is invariant under $\sigma^{\prime}$ and $\mathcal{B}^{*} \cup \rho\left(\mathcal{A}^{*}\right) \cup \sigma^{\prime}\left(\mathcal{A}^{*}\right)$ is invariant under $\rho^{\prime}$.

(b) Suppose $\mathcal{K}^{*} \subseteq \mathcal{A}^{*}$ and $\mathcal{L}^{*} \subseteq \mathcal{B}^{*}$ satisfy analogues of Rh1, Rh2, and Rh3, that is, $\mathcal{K}^{*} \bullet \tau\left(\mathcal{K}^{*}\right)=\mathcal{A}^{*}, \mathcal{L}^{*} \bullet \rho\left(\mathcal{L}^{*}\right)=\mathcal{B}^{*}, \sigma\left(\mathcal{K}^{*} \bullet \mathcal{L}^{*}\right)=\mathcal{K}^{*} \bullet \mathcal{L}^{*}$. Then, $\mathcal{K}^{*} \bullet \mathcal{L}^{*} \bullet \rho \tau\left(\mathcal{K}^{*}\right) \bullet \sigma^{\prime}\left(\mathcal{K}^{*}\right)$ 
is invariant under $\sigma^{\prime}$, and the region $\mathcal{L}^{*} \cup \rho \tau\left(\mathcal{K}^{*}\right) \cup \sigma^{\prime}\left(\mathcal{K}^{*}\right)$ and its image under $\rho^{\prime}$ partition $\mathcal{B}^{*} \cup \rho\left(\mathcal{A}^{*}\right) \cup \sigma^{\prime}\left(\mathcal{A}^{*}\right)$.

We have seen how one can directly construct $\mathcal{K}$ and $\mathcal{L}$ when $\frac{a}{b}$ is an integer. For noninteger values of $\frac{a}{b}$, we will construct $\mathcal{K}$ and $\mathcal{L}$ in a finite or countable number of steps. In particular, we will show that there are closed regions $\mathcal{A}_{i}^{*}, \mathcal{B}_{i}^{*}, \mathcal{K}_{i}^{*}, \mathcal{L}_{i}^{*}$ for $i \in \mathbb{N}$ with the following properties.

D1. For every $i$,

a. $\tau\left(\mathcal{A}_{i}^{*}\right)=\mathcal{A}_{i}^{*}, \rho\left(\mathcal{B}_{i}^{*}\right)=\mathcal{B}_{i}^{*}, \sigma\left(\mathcal{A}_{i}^{*} \bullet \mathcal{B}_{i}^{*}\right)=\mathcal{A}_{i}^{*} \bullet \mathcal{B}_{i}^{*}$.

b. $\mathcal{K}_{i}^{*} \subseteq \mathcal{A}_{i}^{*}$ and $\mathcal{L}_{i}^{*} \subseteq \mathcal{B}_{i}^{*}$, and they satisfy analogues of Rh1, Rh2, and Rh3.

D2. $\mathcal{A}=\operatorname{cl}\left(\bigcup_{i} \mathcal{A}_{i}^{*}\right)$ and $\mathcal{B}=\operatorname{cl}\left(\bigcup_{i} \mathcal{B}_{i}^{*}\right)$.

If so, let $\mathcal{K}:=\operatorname{cl}\left(\bigcup_{i} \mathcal{K}_{i}^{*}\right) \subseteq \mathcal{A}$, and $\mathcal{L}:=\operatorname{cl}\left(\bigcup_{i} \mathcal{L}_{i}^{*}\right) \subseteq \mathcal{B}$. Then if in addition, the unions $\mathcal{K} \cup \tau(\mathcal{K})$ and $\mathcal{L} \cup \rho(\mathcal{L})$ are disjoint up to sets of measure zero, then $\mathcal{K}$ and $\mathcal{L}$ satisfy Rh1, Rh2, and Rh3.

Set $a_{1}=a, b_{1}=b$. Suppose $1<\frac{a_{1}}{b_{1}}<2$, that is, $\mathcal{A}$ is larger than $\mathcal{B}$. Let $v_{1}=b_{1}-\left(a_{1}-b_{1}\right)=$ $2 b_{1}-a_{1}$ and $q_{1}=\left\lfloor\frac{b_{1}}{v_{1}}\right\rfloor$. Recall that $\mathcal{A}=[0, b] \times[0, b]$, and $\mathcal{B}=[b-a, 0] \times[0, b]$. Consider all the squares of the form $\left[v_{1} i_{1}, v_{1}\left(i_{1}+1\right)\right] \times\left[b_{1}-v_{1}\left(j_{1}+1\right), b_{1}-v_{1} j_{1}\right]$, where $i_{1}$ and $j_{1}$ are nonnegative integers such that $i_{1}+j_{1} \leq q_{1}-1$. See for example Figure $6(\mathrm{a})$, where $q_{1}=3$. The squares all have edge length $v_{1}$, and they form a triangular array of squares propped against the left and upper edges of $\mathcal{A}$. We say that the array is anchored at the upper-left vertex of $\mathcal{A}$, and is of order $q_{1}$, with edge length $v_{1}$. If in addition, $q_{1}>1$, construct a second array of squares anchored at the lower-right vertex of $\mathcal{A}$ of order $q_{1}-1$ with edge length $v_{1}$. This is the largest order of a second array that can be constructed such that the two arrays do not overlap. The union $\mathcal{A}_{1}^{*}$ of the two arrays is invariant under $\tau$. Next, form two arrays within $\mathcal{B}$, both of order $q_{1}-1$ and edge length $v_{1}$, one anchored at the upper-right vertex, and another at the lower-left vertex of $\mathcal{B}$. Let $\mathcal{B}_{1}^{*}$ be the union of these two arrays. Then $\rho\left(\mathcal{B}_{1}^{*}\right)=\mathcal{B}_{1}^{*}$ and $\sigma\left(\mathcal{A}_{1}^{*} \cup \mathcal{B}_{1}^{*}\right)=\mathcal{A}_{1}^{*} \cup \mathcal{B}_{1}^{*}$, so that $\mathcal{A}_{1}^{*}$ and $\mathcal{B}_{1}^{*}$ satisfy D1a.

Suppose $2<\frac{a_{1}}{b_{1}}<3$. This time, $\mathcal{B}$ is larger than $\mathcal{A}$ and we set $v_{1}=\left(a_{1}-b_{1}\right)-b_{1}=a_{1}-2 b_{1}$ and $q_{1}=\left\lfloor\frac{b_{1}}{v_{1}}\right\rfloor$. See Figure $6(\mathrm{~b})$. Within $\mathcal{A}$, construct two arrays of edge length $v_{1}$, one with order $q_{1}$ anchored at the lower-right vertex of $\mathcal{A}$, and the other with order $q_{1}-1$ (if positive) anchored at the upper-left vertex of $\mathcal{A}$. We denote the union of these arrays by $\mathcal{A}_{1}^{*}$. Then form two arrays within $\mathcal{B}$, both of order $q_{1}$ and edge length $v_{1}$, one anchored at the upper-right vertex of $\mathcal{B}$, and another at the lower-left vertex of $\mathcal{B}$. Let $\mathcal{B}_{1}^{*}$ be the union of these two arrays. Again, D1a is satisfied.

To construct $\mathcal{K}_{1}^{*}$ and $\mathcal{L}_{1}^{*}$, draw the diagonals of each square in each array, thereby dividing each square into four congruent triangles (as done in the odd-integer-ratio case). Consider the arrays in $\mathcal{A}_{1}$ and $\mathcal{B}_{1}$ anchored at a shared vertex of $\mathcal{A}$ and $\mathcal{B}$. For each square in these arrays, color the upper and lower triangles red. For the remaining two arrays, 


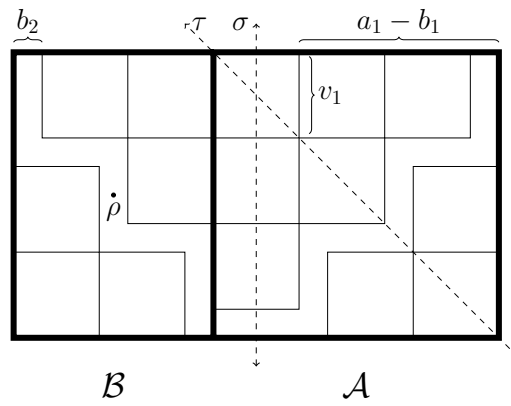

(a)

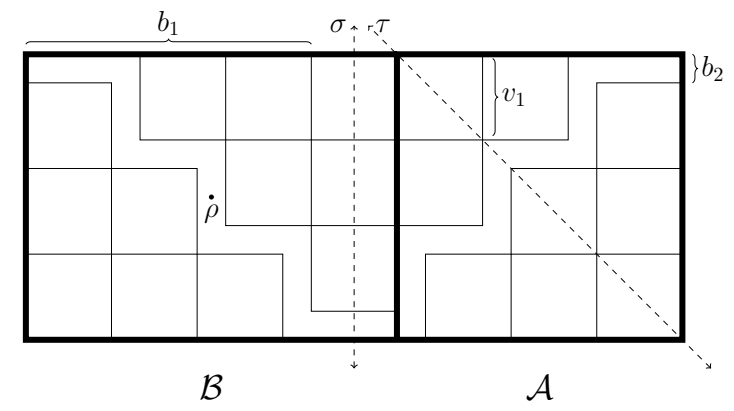

(b)

Figure 6: First steps in constructing $\mathcal{K}$ and $\mathcal{L}$ for (a) $1<\frac{a}{b}<2$ and (b) $2<\frac{a}{b}<3$.

color the left and right triangles of each square red as in Figure 7 . Let $\mathcal{K}_{1}^{*}$ and $\mathcal{L}_{1}^{*}$ be the union of the red regions contained in $\mathcal{A}_{1}^{*}$ and $\mathcal{B}_{1}^{*}$, respectively. By construction, $\mathcal{K}_{1}^{*}$ and $\mathcal{L}_{1}^{*}$ satisfy D1b.

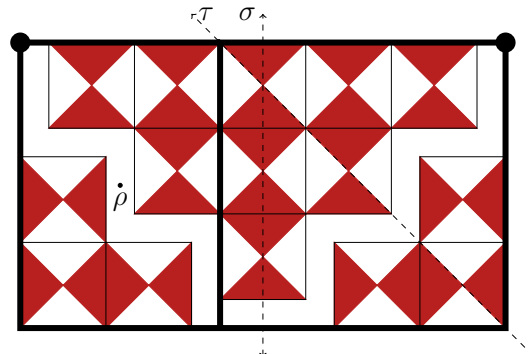

(a)

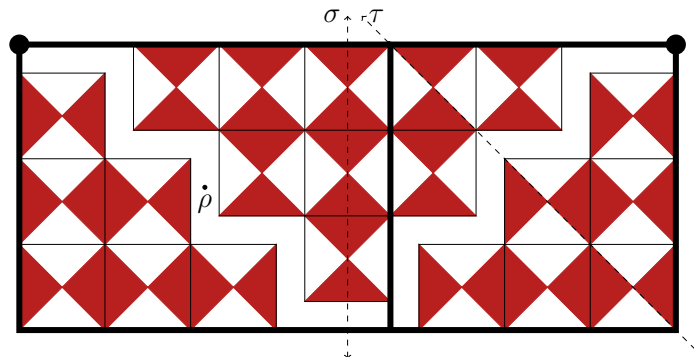

(b)

Figure 7: Subregions $\mathcal{K}_{1}^{*}$ and $\mathcal{L}_{1}^{*}$ of $\mathcal{A}_{1}^{*}$ and $\mathcal{B}_{1}^{*}$ for (a) $1<\frac{a}{b}<2$ and (b) $2<\frac{a}{b}<3$.

Let $b_{2}=b_{1}-v_{1} q_{1}$. This is the uniform width of the "path" $\mathcal{S}_{1}$ left uncovered by $\mathcal{A}_{1}^{*}$ and $\mathcal{B}_{1}^{*}$ in $\mathcal{R}$. Thus, if $\frac{b_{1}}{v_{1}}$ is an integer, then $\mathcal{A}=\mathcal{A}_{1}^{*}$ and $\mathcal{B}=\mathcal{B}_{1}^{*}$, and so we are done if we set $\mathcal{K}=\mathcal{K}_{1}^{*}$ and $\mathcal{L}=\mathcal{L}_{1}^{*}$. Otherwise, consider the central column of squares, that is, the column fixed by $\sigma$. The squares in this column cover a vertical strip of $\mathcal{R}$ of width $v_{1}$ except for a rectangle $\hat{\mathcal{R}}$ with dimensions $b_{2} \times v_{1}$, where $b_{2}<v_{1}$. Partition $\hat{\mathcal{R}}$ into a square $\hat{\mathcal{A}}$ of edge length $b_{2}$ and a rectangle $\hat{\mathcal{B}}$ as in Figure 8 . Let $\hat{\rho}$ be the half-turn about the center of $\hat{\mathcal{B}}$ and $\hat{\tau}$ be the reflection in the line containing the diagonal of $\hat{\mathcal{A}}$ that passes through one corner of the central column of squares.

Lemma 7. It suffices to find $\hat{\mathcal{K}} \subseteq \hat{\mathcal{A}}$ and $\hat{\mathcal{L}} \subseteq \hat{\mathcal{B}}$ that satisfy the corresponding analogues of Rh1, Rh2, and Rh3.

Proof of Lemma \%. Suppose $1<\frac{a_{1}}{b_{1}}<2$. Then, $\hat{\mathcal{R}} \subseteq \mathcal{A}$. Consider the sequences $\left\{R_{j}\right\}$ and 


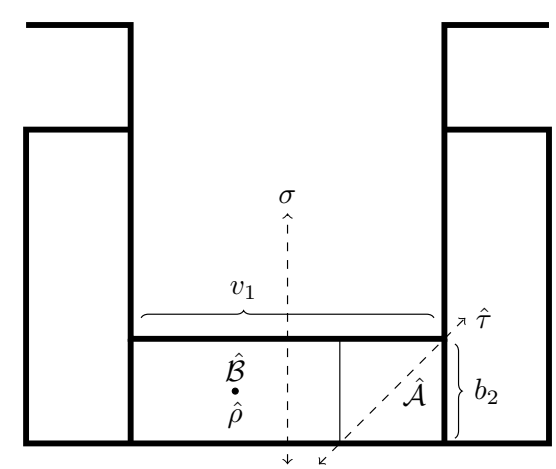

Figure 8: The rectangle $\hat{\mathcal{R}}=\hat{\mathcal{A}} \bullet \hat{\mathcal{B}}$.

$\left\{F_{j}\right\}$ defined by $R_{1}=\hat{\mathcal{R}}=\hat{\mathcal{A}} \bullet \hat{\mathcal{B}}, F_{1}=\hat{\mathcal{K}} \bullet \hat{\mathcal{L}}$, and for $1 \leq j \leq 4 q_{1}$,

$$
R_{j+1}=\left\{\begin{array}{rr}
\tau\left(R_{j}\right), & j \equiv 1(\bmod 4) \\
\sigma\left(R_{j}\right), & j \equiv 0(\bmod 2) \\
\rho\left(R_{j}\right), & j \equiv 3(\bmod 4)
\end{array} \text { and } F_{j+1}=\left\{\begin{aligned}
\tau\left(\operatorname{cl}\left(R_{j} \backslash F_{j}\right)\right), & j \equiv 1(\bmod 4) \\
\sigma\left(F_{j}\right), & j \equiv 0(\bmod 2) \\
\rho\left(\operatorname{cl}\left(R_{j} \backslash F_{j}\right)\right), & j \equiv 3(\bmod 4)
\end{aligned}\right.\right.
$$

The rectangles $R_{j}$ generated are labeled in Figure 9 by their indices. Note that the orientations of the last rectangles in the sequence are not necessarily the same as those in the figure. The longer edges of $R_{4 q_{1}-3}$ and $R_{4 q_{1}-2}$ may be horizontal and vertical, respectively. Regardless of the orientations, the following properties hold.

(a) For $0 \leq k \leq q_{1}-1, R_{4 k+1}$ and $R_{4 k+2}$ are non-overlapping $\tau$-images of each other, and $F_{4 k+1} \bullet F_{4 k+2}$ and its image under $\tau$ partition $R_{4 k+1} \bullet R_{4 k+2}$.

(b) For $0 \leq k \leq q_{1}-2, R_{4 k+3}$ and $R_{4 k+4}$ are non-overlapping $\rho$-images of each other, and $F_{4 k+3} \cup F_{4 k+4}$ and its image under $\rho$ partition $R_{4 k+3} \cup R_{4 k+4}$.

(c) The regions $R_{4 q_{1}-1}$ and $R_{4 q_{1}}$ overlap on a rectangle congruent to $\hat{\mathcal{B}}$. By the choice of $\hat{\mathcal{L}}$, the portions of $F_{4 q_{1}-1}$ and $F_{4 q_{1}}$ inside this rectangle coincide completely.

(d) For $1 \leq k \leq 2 q_{1}, R_{2 k}$ and $R_{2 k+1}$ are $\sigma$-images of each other. The same holds true for $F_{2 k}$ and $F_{2 k+1}$.

(e) The union $\bigcup_{j=1}^{4 q_{1}} R_{j}$ covers $\mathcal{R} \backslash\left(\mathcal{A}_{1}^{*} \cup \mathcal{B}_{1}^{*}\right)$ except for a square region $\mathcal{H}$. The rectangle $R_{4 q_{1}+1}$ covers this. By the choice of $\hat{\mathcal{K}}$, the portion of $F_{4 q_{1}+1}$ inside this square and the image of this portion under $\tau$ partition the square.

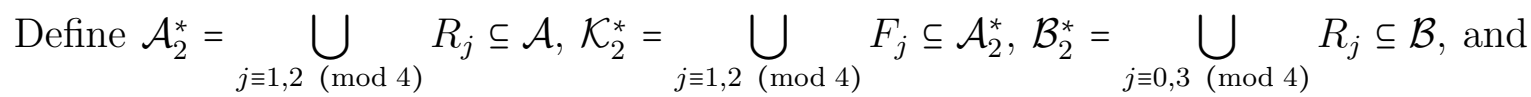
$\mathcal{L}_{2}^{*}=\bigcup_{j=0,3} F_{(\bmod 4)} \subseteq \mathcal{B}_{2}^{*}$. We see that these, with $\tau, \rho$, and $\sigma$, satisfy analogues of Rh1, Rh2, and Rh3. Moreover, $\mathcal{A}_{i}^{*}, \mathcal{B}_{i}^{*}, \mathcal{K}_{i}^{*}$, and $\mathcal{L}_{i}^{*}$ for $i=1,2$ satisfy conditions D1 and D2. 


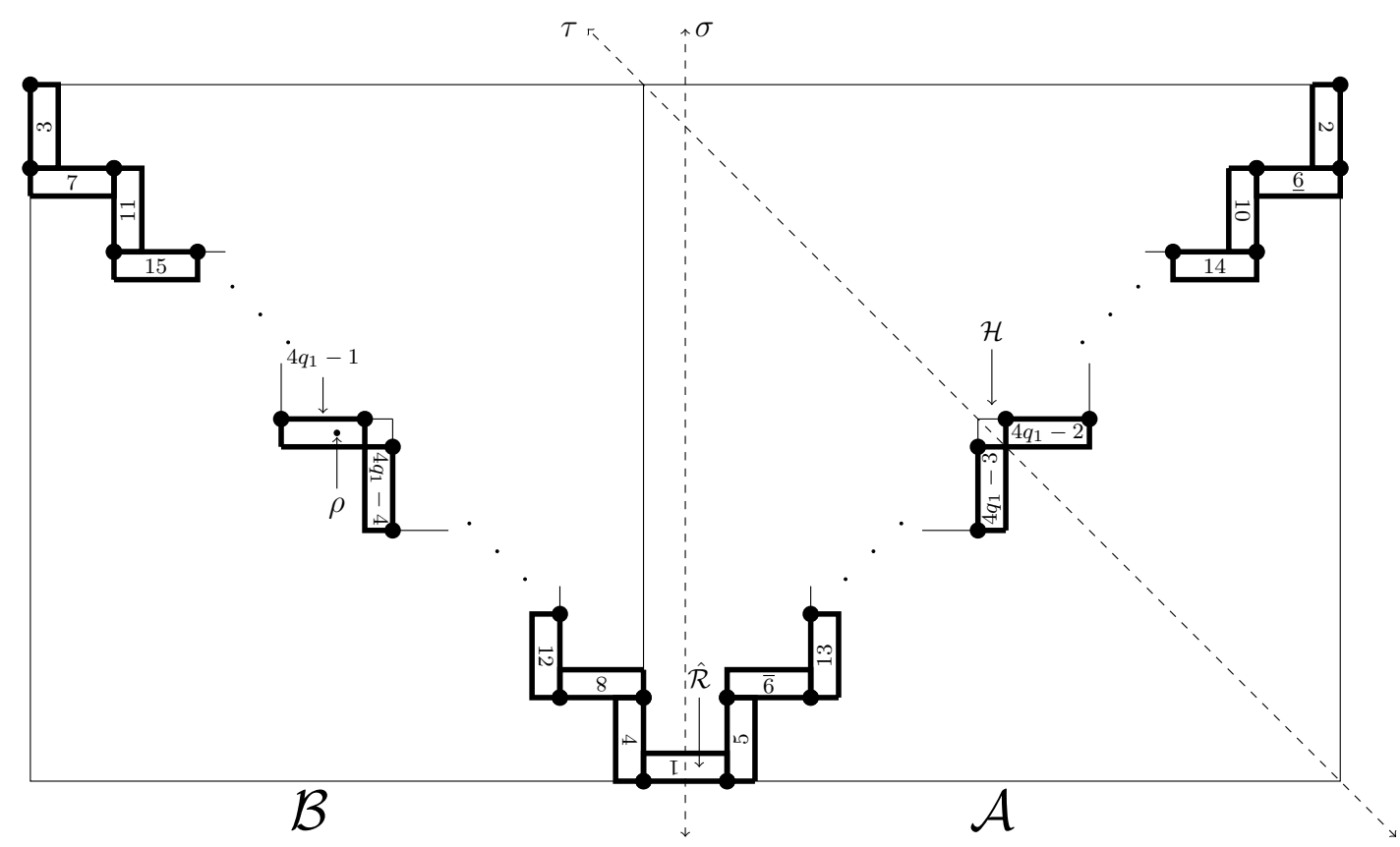

Figure 9: Filling up the uncovered path $\mathcal{S}_{1}$ in $\mathcal{R}$ by applying a sequence of $\sigma, \tau$, and $\rho$ on $\hat{\mathcal{R}}$. The two vertices of rectangle $R_{i}$ marked by big dots are the corresponding images of the two vertices marked in $R_{i-1}$.

The case when $2<\frac{a_{1}}{b_{1}}<3$ is treated analogously. In this case, $\hat{\mathcal{R}} \subseteq \mathcal{B}$. Define the sequences $\left\{R_{j}\right\}$ and $\left\{F_{j}\right\}$ by $R_{1}=\hat{\mathcal{R}}=\hat{\mathcal{A}} \bullet \hat{\mathcal{B}}, F_{1}=\hat{\mathcal{K}} \bullet \hat{\mathcal{L}}$, and for $1 \leq j \leq 4 q_{1}+2$,

$$
R_{j+1}=\left\{\begin{array}{lll}
\rho\left(R_{j}\right), & j \equiv 1 & (\bmod 4) \\
\sigma\left(R_{j}\right), & j \equiv 0 & (\bmod 2) \\
\tau\left(R_{j}\right), & j \equiv 3 & (\bmod 4)
\end{array} \text { and } F_{j+1}=\left\{\begin{aligned}
\rho\left(\operatorname{cl}\left(R_{j} \backslash F_{j}\right)\right), & j \equiv 1(\bmod 4) \\
\sigma\left(F_{j}\right), & j \equiv 0(\bmod 2) \\
\tau\left(\operatorname{cl}\left(R_{j} \backslash F_{j}\right)\right), & j \equiv 3(\bmod 4) .
\end{aligned}\right.\right.
$$

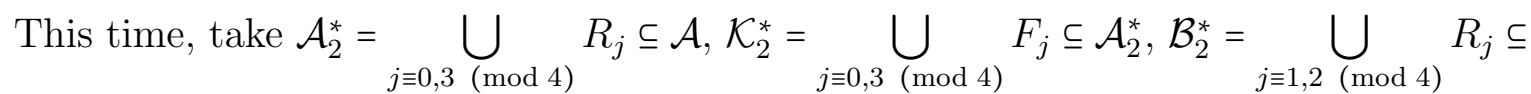
$\mathcal{B}$, and $\mathcal{L}_{2}^{*}=\bigcup_{j \equiv 1,2} F_{(\bmod 4)} F_{j} \subseteq \mathcal{B}_{2}^{*}$.

Remark 8. As in Lemma 4, the properties enumerated in the proof of Lemma 7 are true for suitably chosen subsets of $\hat{\mathcal{A}}$ and $\hat{\mathcal{B}}$. In particular, suppose $\hat{\mathcal{A}}^{*} \subseteq \hat{\mathcal{A}}$ and $\hat{\mathcal{B}}^{*} \subseteq \hat{\mathcal{B}}$ such that $\hat{\tau}\left(\hat{\mathcal{A}}^{*}\right)=\hat{\mathcal{A}}^{*}, \hat{\rho}\left(\hat{\mathcal{B}}^{*}\right)=\hat{\mathcal{B}}^{*}, \sigma\left(\hat{\mathcal{A}}^{*} \sqcup \hat{\mathcal{B}}^{*}\right)=\hat{\mathcal{A}}^{*} \sqcup \hat{\mathcal{B}}^{*}$. Suppose $\hat{\mathcal{K}}^{*} \subseteq \hat{\mathcal{A}}^{*}$ and $\hat{\mathcal{L}}^{*} \subseteq \hat{\mathcal{B}}^{*}$ satisfy analogues of Rh1, Rh2, and Rh3. Obtain the sequences $\left\{R_{j}^{*}\right\}$ and $\left\{F_{j}^{*}\right\}$ analogously, and form $\hat{\mathcal{A}}_{2}^{\star}, \hat{\mathcal{B}}_{2}^{\star}, \hat{\mathcal{K}}_{2}^{\star}, \hat{\mathcal{L}}_{2}^{\star}$ accordingly. Then, these also satisfy analogues of Rh1, Rh2, and Rh3. 
We thus have the following procedure.

\section{Step 1:}

- Without loss of generality, assume that the rectangle $\mathcal{R}_{1}:=\mathcal{R}$ has a non-integer edge ratio between 1 and 3. Otherwise we can use Lemma 3 or Lemma 4.

- Fill $\mathcal{R}_{1}$ with $\mathcal{A}_{1}^{*}, \mathcal{B}_{1}^{*}, \mathcal{K}_{1}^{*}$, and $\mathcal{L}_{1}^{*}$ as described above, leaving the unfilled path $\mathcal{S}_{1}$, with central rectangle $\mathcal{R}_{2}$.

\section{Step 2:}

- Without loss of generality, assume that the central rectangle $\mathcal{R}_{2}$ in $\hat{\mathcal{S}}_{1}:=\mathcal{S}_{1}$ has a non-integer edge ratio between 1 and 3. Otherwise we can use Lemma 3 or Lemma 4.

- Fill $\mathcal{R}_{2}$ with $\hat{\mathcal{A}}_{2}^{\star}, \hat{\mathcal{B}}_{2}^{\star}, \hat{\mathcal{K}}_{2}^{*}$, and $\hat{\mathcal{L}}_{2}^{\star}$ as described above, leaving the unfilled path $\hat{\mathcal{S}}_{2}$.

- Fill portions of $\mathcal{S}_{1}$ using the sequence defined in the proof of Lemma 7 to create $\mathcal{A}_{2}^{*}$, $\mathcal{B}_{2}^{*}, \mathcal{K}_{2}^{*}$, and $\mathcal{L}_{2}^{*}$, leaving copies of $\hat{\mathcal{S}}_{2}$. The big dots in Figure 9 are at the ends of the aforementioned copies of $\hat{\mathcal{S}}_{2}$, thus the union $\mathcal{S}_{2}$ of these copies is a connected unfilled path.

Step $\boldsymbol{n}, \boldsymbol{n} \geq 3$ : This is treated analogously as Step 2 .

- Without loss of generality, assume that the central rectangle $\mathcal{R}_{n}$ in $\hat{\mathcal{S}}_{n-1}$ has a noninteger edge ratio between 1 and 3. Otherwise we can use Lemma 3 or Lemma 4.

- Fill $\mathcal{R}_{n}$ with $\hat{\mathcal{A}}_{n}^{*}, \hat{\mathcal{B}}_{n}^{*}, \hat{\mathcal{K}}_{n}^{*}$, and $\hat{\mathcal{L}}_{n}^{*}$ as described above, leaving the unfilled path $\hat{\mathcal{S}}_{n}$.

- Fill portions of $\mathcal{S}_{n-1}$ using the corresponding sequences of those in the proof of Lemma 7 , filling out portions of, in order, $\hat{\mathcal{S}}_{n-1}, \hat{\mathcal{S}}_{n-2}, \ldots, \hat{\mathcal{S}}_{2}$, and $\hat{\mathcal{S}}_{1}:=\mathcal{S}_{1}$, to create $\mathcal{A}_{n}^{*}, \mathcal{B}_{n}^{*}, \mathcal{K}_{n}^{*}$, and $\mathcal{L}_{n}^{*}$. Arguing as before, this leaves an empty path $\mathcal{S}_{n}$ which is composed of copies of $\hat{\mathcal{S}}_{n}$.

If $\frac{a}{b}$ is irrational, it is clear that the procedure has infinitely many steps. Note that if $\frac{a}{b}=\frac{a_{1}}{b_{1}}$ is rational, then the ratios $\frac{b_{1}}{v_{1}}=\frac{b_{1}}{\left|2 b_{1}-a_{1}\right|}$ and $\frac{b_{2}}{v_{1}}=\frac{b_{1}}{v_{1}}-q_{1}$ are also rational. In the next step, we construct arrays for a rectangle with edge ratio $\frac{a_{2}}{b_{2}}$ which differs from $\frac{v_{1}}{b_{2}}$ by an integer. It follows that the edge ratio of a rectangle in each succeeding step is rational. Furthermore, the divisor $v_{n}$ used at step $n$ is smaller than the remainder $b_{n}$ of the preceding step, so that the sequence of divisors terminates faster than that of the Euclidean algorithm applied to $a$ and $b$. Thus, there are only finitely many steps in this case.

It is clear from the construction that condition D1 is satisfied. Note also that at each step, the squares used to pack the rectangle $\mathcal{R}$ overlap with squares used in previous steps only along sets of measure zero. Thus, $\bigcup_{i} \mathcal{A}_{i}^{*}$ and $\bigcup_{i} \mathcal{B}_{i}^{*}$ are disjoint unions. Furthermore, because the width of the unfilled path approaches zero, indeed, condition D2 is satisfied. In fact, if $\frac{a}{b}$ is rational, then the width and area of this path becomes zero after a finite 
number of steps. This implies that the unions in condition D2 are finite unions, and it follows that $\mathcal{K} \cup \tau(\mathcal{K})$ and $\mathcal{L} \cup \rho(\mathcal{L})$ are disjoint unions up to sets of measure zero. It remains to show that this last condition also holds if $\frac{a}{b}$ is irrational.

Note that for each $t \in \mathbb{N}$, the path that remains unfilled after step $t$ consists of $\operatorname{cl}\left(\mathcal{A} \backslash \bigcup_{i=1}^{t} \mathcal{A}_{i}^{*}\right)$ and $\operatorname{cl}\left(\mathcal{B} \backslash \bigcup_{i=1}^{t} \mathcal{B}_{i}^{*}\right)$. Moreover, we obtain that $\mathcal{A}_{t+1}^{*} \subseteq \operatorname{cl}\left(\mathcal{A} \backslash \bigcup_{i=1}^{t} \mathcal{A}_{i}^{*}\right)$ and $\mathcal{B}_{t+1}^{*} \subseteq \operatorname{cl}\left(\mathcal{B} \backslash \bigcup_{i=1}^{t} \mathcal{B}_{i}^{*}\right)$. Thus, $\mathcal{K}=\operatorname{cl}\left(\bigcup_{i} \mathcal{K}_{i}^{*}\right)=\biguplus_{i=1}^{t} \mathcal{K}_{i}^{*} \cup \operatorname{cl}\left(\biguplus_{i=t+1}^{\infty} \mathcal{K}_{i}^{*}\right)$ and $\mathcal{L}=\operatorname{cl}\left(\bigcup_{i} \mathcal{L}_{i}^{*}\right)=$ $\biguplus_{i=1}^{t} \mathcal{L}_{i}^{*} \cup \operatorname{cl}\left(\biguplus_{i=t+1}^{\infty} \mathcal{L}_{i}^{*}\right)$, where $\operatorname{cl}\left(\biguplus_{i=t+1}^{\infty} \mathcal{K}_{i}^{*}\right) \subseteq \operatorname{cl}\left(\mathcal{A} \backslash \bigcup_{i=1}^{t} \mathcal{A}_{i}^{*}\right)$ and $\operatorname{cl}\left(\biguplus_{i=t+1}^{\infty} \mathcal{L}_{i}^{*}\right) \subseteq \operatorname{cl}\left(\mathcal{B} \backslash \biguplus_{i=1}^{t} \mathcal{B}_{i}^{*}\right)$. Because of condition D1b, it suffices to show that the measure of the unfilled path approaches zero to justify why $\mathcal{K} \cup \tau(\mathcal{K})$ and $\mathcal{L} \cup \rho(\mathcal{L})$ are disjoint unions up to sets of measure zero.

Consider first the ratio of the measure of $\mathcal{A}_{1}^{*} \cup \mathcal{B}_{1}^{*}$ to the measure of $\mathcal{R}$.

Case 1: Suppose $1<\frac{a_{1}}{b_{1}}<2$. In this case, $q_{1}<\frac{b_{1}}{2 b_{1}-a_{1}}<q_{1}+1$, and so $\frac{q_{1}+1}{2 q_{1}+1}<\frac{b_{1}}{a_{1}}<\frac{q_{1}}{2 q_{1}-1}$. From the construction, the aforementioned ratio of measures is equal to

$$
q_{1}\left(2 q_{1}-1\right) \cdot \frac{\left(2 b_{1}-a_{1}\right)^{2}}{a_{1} b_{1}}=q_{1}\left(2 q_{1}-1\right)\left(\frac{2 b_{1}-a_{1}}{b_{1}}\right)^{2} \cdot \frac{b_{1}}{a_{1}}>\frac{q_{1}\left(2 q_{1}-1\right)}{\left(q_{1}+1\right)\left(2 q_{1}+1\right)}>\frac{1}{6} .
$$

Case 2: Suppose $2<\frac{a_{1}}{b_{1}}<3$. In this case, $q_{1}<\frac{b_{1}}{a_{1}-2 b_{1}}<q_{1}+1$, and so $\frac{q_{1}}{2 q_{1}+1}<\frac{b_{1}}{a_{1}}<\frac{q_{1}+1}{2 q_{1}+3}$. From the construction, the aforementioned ratio of measures is equal to

$$
q_{1}\left(2 q_{1}+1\right) \cdot \frac{\left(a_{1}-2 b_{1}\right)^{2}}{a_{1} b_{1}}=q_{1}\left(2 q_{1}+1\right)\left(\frac{a_{1}-2 b_{1}}{b_{1}}\right)^{2} \cdot \frac{b_{1}}{a_{1}}>\left(\frac{q_{1}}{q_{1}+1}\right)^{2}>\frac{1}{4} .
$$

We note that in the procedure outlined in the proof of Lemma 7, each of the last two rectangles overlap with some previous rectangle except on a square region congruent to $\hat{\mathcal{A}}$. Thus, we also look for a lower bound for the ratio of the measure of $\mathcal{A}_{1}^{*}$ to that of $\mathcal{A}$. In either of the two cases above, this ratio is

$$
q_{1}^{2} \cdot \frac{\left|2 b_{1}-a_{1}\right|^{2}}{b_{1}^{2}}>\left(\frac{q_{1}}{q_{1}+1}\right)^{2}>\frac{1}{4} .
$$

These computations show that at each step of the construction, the ratio of the measure of the portion that will remain unfilled is at most $\frac{5}{6}$ of the measure of the unfilled portion in the previous step. Thus, the measure of the unfilled portion approaches zero as the construction is carried out. It follows that $\mathcal{K}$ and $\mathcal{L}$ satisfy Rh1, Rh2, and Rh3.

Forming $\mathcal{S}$ as in Figure 1 and $\mathcal{E}=P(\Gamma)(\mathcal{K} \cup \mathcal{L})$, we have that $F=\operatorname{cl}(\mathcal{S} \cup \mathcal{E})$ is a compact fundamental domain for $\Gamma$ with $[S(F): P(\Gamma)]=2$. This completes the proof of Theorem 1.

In Figure 10, we show a portion of the tiling by the fundamental domain for the rhombic lattice $\Gamma$ with basis $\left\{\left(\begin{array}{c}22 \\ 0\end{array}\right),\left(\begin{array}{l}11 \\ 25\end{array}\right)\right\}$ generated by the procedure described. 


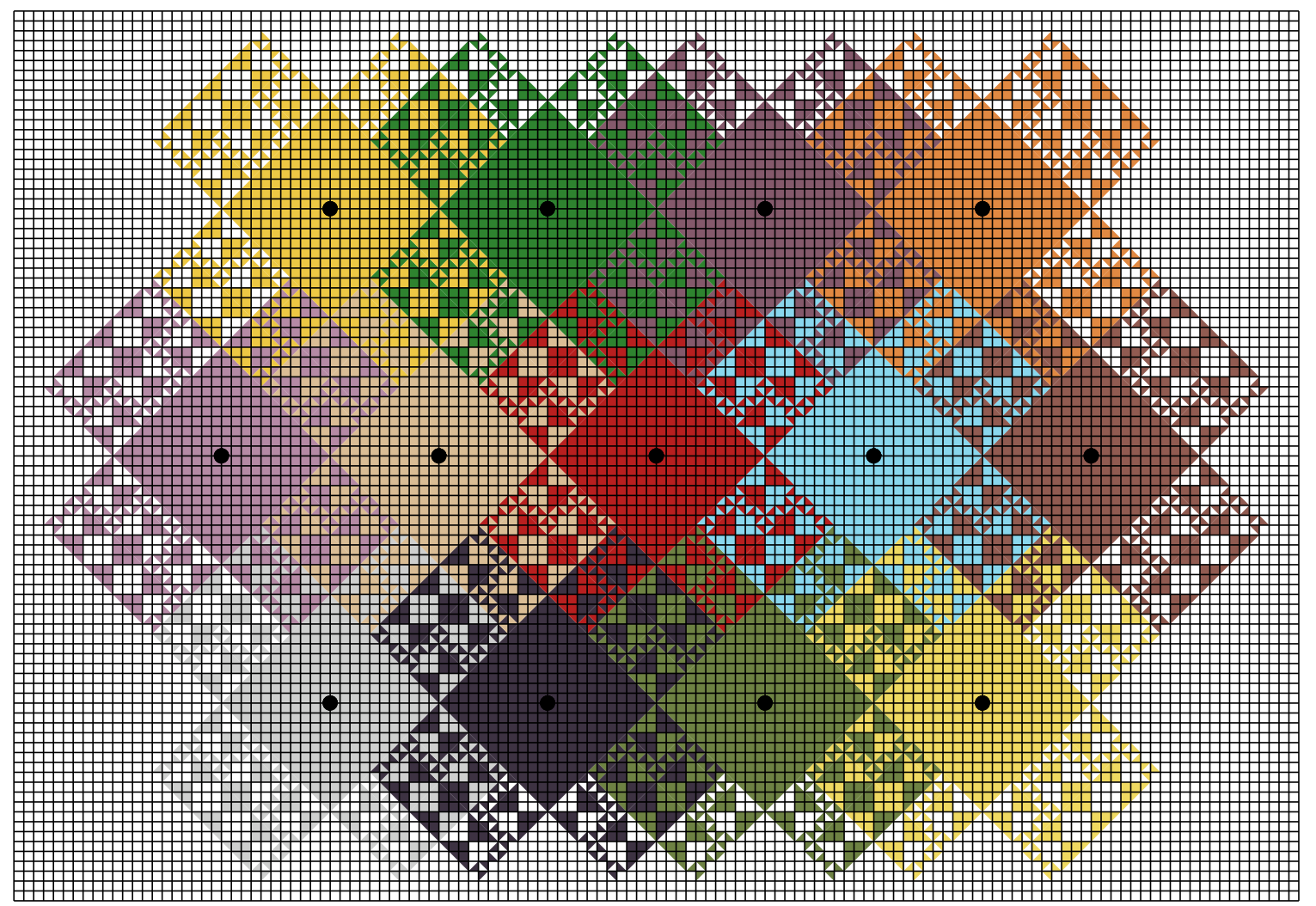

Figure 10: Fundamental domain for the rhombic lattice $\Gamma$ with $m=11, n=25$, constructed using the procedure, together with some of its $\Gamma$-translates.

\section{Acknowledgements}

The authors would like to thank the anonymous referee for valuable remarks that improved the quality of the paper. J.R.C.G. Damasco is grateful to the University of the Philippines System for financial support through its Faculty, REPS, and Administrative Staff Development Program. D. Frettlöh is grateful to the Research Center for Mathematical Modelling, Bielefeld University, and the University of the Philippines System for financial support.

\section{References}

[1] M. Baake, R. Klitzing, M. Schlottmann. Fractally shaped acceptance domains of quasiperiodic square-triangle tilings with dodecagonal symmetry. Physica A, 191:554$558,1992$.

[2] E. Cockayne. Nonconnected atomic surfaces for quasicrystalline sphere packings. Phys. Rev. B, 49:5896-5910, 1994. 
[3] J. Damasco, D. Frettlöh, M. Loquias. Highly symmetric fundamental domains for lattices in $\mathbb{R}^{2}$ and $\mathbb{R}^{3}$. arXiv:1305.1798.

[4] M. Dehn. Über Zerlegung von Rechtecken in Rechtecke. Math. Annalen, 57:314-332, 1903.

[5] V. Elser. Exceptionally symmetric fundamental domains for the root lattices in 2D. In Aperiodic Order, Tagungsbericht Oberwolfach, No. 20, Mathematisches Forschungsinstitut Oberwolfach, Germany, 2001. 6.

[6] V. Elser. Fractal Fun. http://uuuuuu. lassp. cornell.edu/gallery/fractal_fun, accessed 05 January 2018. 